\title{
CONNECTEDNESS EXTENSIONS FOR ABELIAN VARIETIES
}

\author{
A. SILVERBERG* AND YU. G. ZARHIN**
}

\section{INTRODUCTION}

Suppose $A$ is an abelian variety defined over a field $F, \ell$ is a prime number, and $\ell \neq \operatorname{char}(F)$. Let $F^{s}$ denote a separable closure of $F$, let $T_{\ell}(A)=\lim _{\leftarrow} A_{\ell^{r}}$ (the Tate module), let $V_{\ell}(A)=T_{\ell}(A) \otimes \mathbf{z}_{\ell} \mathbf{Q}_{\ell}$, and let $\rho_{A, \ell}$ denote the $\ell$-adic representation

$$
\rho_{A, \ell}: \operatorname{Gal}\left(F^{s} / F\right) \rightarrow \operatorname{Aut}\left(T_{\ell}(A)\right) \subseteq \operatorname{Aut}\left(V_{\ell}(A)\right) .
$$

If $L$ is an extension of $F$ in $F^{s}$, let $G_{L, A}$ denote the image of $\operatorname{Gal}\left(F^{s} / L\right)$ under $\rho_{A, \ell}$. Let $\mathfrak{G}_{\ell}(F, A)$ denote the algebraic envelope of the image of $\rho_{A, \ell}$, i.e., the Zariski closure of $G_{F, A}$ in $\operatorname{Aut}\left(V_{\ell}(A)\right) \cong \mathrm{GL}_{2 d}\left(\mathbf{Q}_{\ell}\right)$, where $d=\operatorname{dim}(A)$.

Let $F_{\Phi, \ell}(A)$ be the smallest extension $F^{\prime}$ of $F$ such that $\mathfrak{G}_{\ell}\left(F^{\prime}, A\right)$ is connected. We call this extension the $\ell$-connectedness extension, or connectedness extension.

The algebraic group $\mathfrak{G}_{\ell}(F, A)$ and the field $F_{\Phi, \ell}(A)$ were introduced by Serre (15], 16], 17]), who proved that if $F$ is a global field or a finitely generated extension of $\mathbf{Q}$, then $F_{\Phi, \ell}(A)$ is independent of $\ell$ (see also [6], [7], [8]). In such cases, we will denote the field $F_{\Phi, \ell}(A)$ by $F_{\Phi}(A)$. For every integer $n \geq 3$ we have

$$
F_{\Phi}(A) \subseteq F\left(A_{n}\right)
$$

(see [2], 迆, Proposition 3.6 of [3], and [23]). Larsen and Pink [8] recently proved that for every integer $n \geq 3$,

$$
F_{\Phi}(A)=\bigcap_{\text {prime } p \geq n} F\left(A_{p}\right) .
$$

In 23 we found conditions for the connectedness of $\mathfrak{G}_{\ell}(F, A)$, while in [24] we used connectedness extensions and Serre's $\ell$-independence results to obtain $\ell$-independence results for the intersection of $\mathfrak{G}_{\ell}(F, A)\left(\mathbf{Q}_{\ell}\right)$ with the torsion subgroup of the center of $\operatorname{End}(A) \otimes \mathbf{Q}$.

Let $F(\operatorname{End}(A))$ denote the smallest extension of $F$ over which all the endomorphisms of $A$ are defined. Then (see Proposition 2.10 of [23]),

$$
F(\operatorname{End}(A)) \subseteq F_{\Phi, \ell}(A) .
$$

Therefore, $\mathfrak{G}_{\ell}(F, A)$ fails to be connected when the ground field is not a field of definition for the endomorphisms of $A$. For example, if $F$ is a subfield of $\mathbf{C}$, and $A$ is an elliptic curve over $F$ with complex multiplication by an imaginary quadratic field $K$ which is not contained in $F$, then $F \neq K F=F(\operatorname{End}(A)) \subseteq F_{\Phi, \ell}(A)$. More generally, if $A$ is an abelian variety of CM-type, and $\tilde{K}$ is the reflex CM-field, then $F(\operatorname{End}(A)) \supseteq \tilde{K}$; if $\tilde{K}$ is not contained in $F$ then $F \neq F(\operatorname{End}(A)) \subseteq F_{\Phi, \ell}(A)$. It is

\footnotetext{
* Partially supported by the National Science Foundation.

** Partially supported by the Ohio State University Mathematics Research Institute and the Deutsche Forschungsgemeinschaft.
} 
therefore natural to enlarge the ground field $F$ so that it is a field of definition for the endomorphisms of $A$.

By enlarging the ground field, we may assume that $F=F(\operatorname{End}(A))=F_{\Phi, \ell}(A)$. We then consider the $F$-forms $B$ of $A$ such that $F=F(\operatorname{End}(B))$. For such $B$, we describe the connectedness extensions $F_{\Phi, \ell}(B) / F$ (see $\S 3$, especially Theorem 3.1 and Corollary 3.2). Properties of Mumford-Tate groups given in $\$ 2$ allow us to obtain explicit information about the connectedness extensions $F_{\Phi, \ell}(B) / F$ under additional conditions (see Theorems 3.4 and 3.5). Our conditions in Theorems 3.4 and 3.5 are based on Weil's philosophy in 22.5] whereby exceptional Hodge classes arise from certain abelian varieties that have a CM-field embedded in their endomorphism algebras. In $\S$ t we use the results of $\S 3$ to explicitly compute nontrivial connectedness extensions in special cases.

Acknowledgments: The authors would like to thank the Mathematische Institut der Universität Erlangen-Nürnberg for its hospitality.

\section{Definitions, notation, AND Lemmas}

Let $\mathbf{Z}, \mathbf{Q}$, and $\mathbf{C}$ denote respectively the integers, rational numbers, and complex numbers. If $r$ is an integer, then $\mathbf{Q}(r)$ denotes the rational Hodge structure of weight $-2 r$ on $\mathbf{Q}$ (see $\S 1$ of [4]). If $a$ and $b$ are integers, let $(a, b)$ denote the greatest common divisor of $a$ and $b$. If $F$ is a field, let $F^{s}$ denote a separable closure of $F$ and let $\bar{F}$ denote an algebraic closure of $F$. If $A$ is an abelian variety over a field $F$, write $\operatorname{End}_{F}(A)$ for the set of endomorphisms of $A$ which are defined over $F$, let $\operatorname{End}(A)=\operatorname{End}_{F^{s}}(A)$, and let $\operatorname{End}^{0}(A)=\operatorname{End}(A) \otimes_{\mathbf{z}} \mathbf{Q}$. Let $Z_{A}$ denote the center of $\operatorname{End}(A)$. If $G$ is an algebraic group, let $G^{0}$ denote the identity connected component.

Lemma 2.1 (Lemma 2.7 of [23]). If $A$ is an abelian variety over a field $F, L$ is a finite extension of $F$ in $F^{s}$, and $\ell$ is a prime number, then

$$
\mathfrak{G}_{\ell}(L, A) \subseteq \mathfrak{G}_{\ell}(F, A) \text { and } \mathfrak{G}_{\ell}(L, A)^{0}=\mathfrak{G}_{\ell}(F, A)^{0} .
$$

In particular, if $\mathfrak{G}_{\ell}(F, A)$ is connected, then $\mathfrak{G}_{\ell}(F, A)=\mathfrak{G}_{\ell}(L, A)$.

Lemma 2.2. Suppose $A$ and $B$ are abelian varieties over a field $F, L$ is a finite extension of $F$ in $F^{s}, \ell$ is a prime number, $\ell \neq \operatorname{char}(F), \mathfrak{G}_{\ell}(F, A)$ is connected, and $A$ and $B$ are isomorphic over $L$. Then:

(i) $\mathfrak{G}_{\ell}(F, B)^{0}=\mathfrak{G}_{\ell}(F, A)$, and

(ii) $\mathfrak{G}_{\ell}(L, B)$ is connected, i.e., $F_{\Phi, \ell}(B) \subseteq L$.

Proof. Since $A$ and $B$ are isomorphic over $L$, and $\mathfrak{G}_{\ell}(F, A)$ is connected, we have

$$
\begin{gathered}
\mathfrak{G}_{\ell}(L, B)=\mathfrak{G}_{\ell}(L, A)=\mathfrak{G}_{\ell}(F, A)=\mathfrak{G}_{\ell}(F, A)^{0} \\
=\mathfrak{G}_{\ell}(L, A)^{0}=\mathfrak{G}_{\ell}(L, B)^{0}=\mathfrak{G}_{\ell}(F, B)^{0},
\end{gathered}
$$

using Lemma 2.1. The result follows.

Proposition 2.3. Suppose $A$ and $B$ are abelian varieties over a field $F, L$ is a field extension of $F$ in $F^{s}$, and $f: A \rightarrow B$ is an isomorphism defined over $L$. Suppose that for every $\sigma \in \operatorname{Gal}\left(F^{s} / F\right)$, the element $f^{-1} \sigma(f)$ of $\operatorname{Aut}(A)$ commutes with every element of $\operatorname{End}_{L}(A)$. Then $\operatorname{End}_{F}(A) \cong \operatorname{End}_{F}(B)$. 
Proof. Define an isomorphism $\varphi: \operatorname{End}_{L}(A) \rightarrow \operatorname{End}_{L}(B)$ by $\varphi(\beta)=f \beta f^{-1}$. For every $\beta \in \operatorname{End}_{L}(A)$ and $\sigma \in \operatorname{Gal}\left(F^{s} / F\right)$, we have $f^{-1} \sigma(f) \beta=\beta f^{-1} \sigma(f)$. Therefore, $\sigma\left(f \sigma^{-1}(\beta) f^{-1}\right)=f \beta f^{-1}$. Thus, $\beta \in \operatorname{End}_{F}(A)$ if and only if $f \beta f^{-1} \in \operatorname{End}_{F}(B)$. In other words, the restriction of $\varphi$ to $\operatorname{End}_{F}(A)$ induces an isomorphism onto $\operatorname{End}_{F}(B)$.

As a corollary we have the following result. See also Lemma 5.1 of [22].

Corollary 2.4. Suppose $A$ is an abelian variety over a field $F$. If an element of $H^{1}\left(\operatorname{Gal}\left(F^{s} / F\right), \operatorname{Aut}(A)\right)$ is represented by a cocycle $c$ with values in the center of $\operatorname{End}^{0}(A)$, and $B$ is the twist of $A$ by $c$, then $\operatorname{End}_{F}(A) \cong \operatorname{End}_{F}(B)$.

Proof. The cocycle $c$ defines an isomorphism $f: A \rightarrow B$ such that for every $\sigma \in$ $\operatorname{Gal}\left(F^{s} / F\right), f^{-1} \sigma(f)=c(\sigma)$. We apply Proposition 2.3.

Lemma 2.5. Suppose $A$ is an abelian variety over a field $F, c$ is a cocycle on $\operatorname{Gal}\left(F^{s} / F\right)$ with values in $\operatorname{Aut}(A), B$ is the twist of $A$ by $c$, and $F=F(\operatorname{End}(A))=$ $F(\operatorname{End}(B))$. Then $c$ is a character with values in $Z_{A}^{\times}$, where $Z_{A}$ denotes the center of $\operatorname{End}(A)$.

Proof. Since $\operatorname{Gal}\left(F^{s} / F\right)$ acts trivially on $\operatorname{End}(A)$, the cocycle $c$ is a homomorphism. Let $f: A \rightarrow B$ be the isomorphism induced by $c$. Then $c(\sigma)=f^{-1} \sigma(f)$ for every $\sigma \in \operatorname{Gal}\left(F^{s} / F\right)$. Since $F=F(\operatorname{End}(A))=F(\operatorname{End}(B))$, it easily follows that $c(\sigma) \in Z_{A}$ and $c(\sigma)^{-1} \in Z_{A}$.

Remark 2.6. If an abelian variety $B$ over $F$ is the twist of an abelian variety $A$ by $c \in H^{1}\left(\operatorname{Gal}\left(F^{s} / F\right)\right.$, Aut $\left.(A)\right)$ then one may easily check that the Galois module $B\left(F^{s}\right)$ is the twist by $c$ of the Galois module $A\left(F^{s}\right)$, and therefore the Galois module $V_{\ell}(B)$ is the twist by $c$ of the Galois module $V_{\ell}(A)$.

We define the Mumford-Tate group of a complex abelian variety $A$ (see $\S 2$ of $[13$ ] or $\S 6$ of [26]). If $A$ is a complex abelian variety, let $V=H_{1}(A(\mathbf{C}), \mathbf{Q})$ and consider the Hodge decomposition $V \otimes \mathbf{C}=H_{1}(A(\mathbf{C}), \mathbf{C})=H^{-1,0} \oplus H^{0,-1}$. Define a homomorphism $\mu: \mathbf{G}_{m} \rightarrow G L(V)$ as follows. For $z \in \mathbf{C}$, let $\mu(z)$ be the automorphism of $V \otimes \mathbf{C}$ which is multiplication by $z$ on $H^{-1,0}$ and is the identity on $H^{0,-1}$.

Definition 2.7. The Mumford-Tate group $M T_{A}$ of $A$ is the smallest algebraic subgroup of $G L(V)$, defined over $\mathbf{Q}$, which after extension of scalars to $\mathbf{C}$ contains the image of $\mu$.

It follows from the definition that $M T_{A}$ is connected.

Define a homomorphism $\varphi: \mathbf{G}_{m} \times \mathbf{G}_{m} \rightarrow G L(V)$ as follows. For $z, w \in \mathbf{C}$, let $\varphi(z, w)$ be the automorphism of $V \otimes \mathbf{C}$ which is multiplication by $z$ on $H^{-1,0}$ and is multiplication by $w$ on $H^{0,-1}$. Then $M T_{A}$ can also be defined as the smallest algebraic subgroup of $G L(V)$, defined over $\mathbf{Q}$, which after extension of scalars to $\mathbf{C}$ contains the image of $\varphi$. The equivalence of the definitions follows easily from the fact that $H^{-1,0}$ is the complex conjugate of $H^{0,-1}$. (See $\S 3$ of [14], where $M T_{A}$ is called the Hodge group. See also $\S 6$ of [26].)

If $A$ is an abelian variety over a subfield $F$ of $\mathbf{C}$, we fix an embedding of $\bar{F}$ in C. This gives an identification of $V_{\ell}(A)$ with $H_{1}(A, \mathbf{Q}) \otimes \mathbf{Q}_{\ell}$, and allows us to view $M T_{A} \times \mathbf{Q}_{\ell}$ as a linear $\mathbf{Q}_{\ell}$-algebraic subgroup of $G L\left(V_{\ell}(A)\right)$. Let

$$
M T_{A, \ell}=M T_{A} \times_{\mathbf{Q}} \mathbf{Q}_{\ell} .
$$


Then $M T_{A}\left(\mathbf{Q}_{\ell}\right)=M T_{A, \ell}\left(\mathbf{Q}_{\ell}\right)$. The Mumford-Tate conjecture for abelian varieties (see 14]) may be reformulated as the equality of $\mathbf{Q}_{\ell}$-algebraic groups, $\mathfrak{G}_{\ell}(F, A)^{0}=$ $M T_{A, \ell}$.

Theorem 2.8 (Piatetski-Shapiro [12], Deligne [4], Borovoi [1]). If $A$ is an abelian variety over a finitely generated extension $F$ of $\mathbf{Q}$, then $\mathfrak{G}_{\ell}(\vec{F}, A)^{0} \subseteq M T_{A, \ell}$.

In $\S 3$ it will be helpful to use a slightly different version of the Mumford-Tate group, as defined by Deligne (see p. 43 and pp. 62-63 of 畍). We will denote this group $\widetilde{M T}_{A}$. (See also pp. 466-467 of [9] for a comparison between $M T_{A}$ and $\widetilde{M T}_{A}$.) Letting $V^{*}$ be the dual of $V$, then $T=V^{\otimes p} \otimes\left(V^{*}\right)^{\otimes q} \otimes \mathbf{Q}(r)$ has a Hodge structure of weight $q-p-2 r$. If $\nu \in \mathbf{G}_{m}$, let $\nu$ act on $\mathbf{Q}(1)$ as $\nu^{-1}$, and we obtain a canonical action of $G L(V) \times \mathbf{G}_{m}$ on $T$. (Note that $V^{*} \cong V \otimes \mathbf{Q}(1)$, since $V$ is a polarized Hodge structure of weight -1 .)

Definition 2.9. The group $\widetilde{M T}_{A}$ is the subgroup of $G L(V) \times \mathbf{G}_{m}$ consisting of the elements which fix all rational tensors of bidegree $(0,0)$ belonging to any $T$.

Lemma 2.10 (Proposition 3.4 of 田). The algebraic group $\widetilde{M T}_{A}$ is the smallest algebraic subgroup of $G L(V) \times \mathbf{G}_{m}$ defined over $\mathbf{Q}$ which, after extension of scalars to $\mathbf{C}$, contains the image of $(\mu, \mathrm{id}): \mathbf{G}_{m} \rightarrow G L(V) \times \mathbf{G}_{m}$.

If $F$ is a field and $\ell$ is a prime number different from $\operatorname{char}(F)$, let

$$
\chi_{\ell}: \operatorname{Gal}\left(F^{s} / F\right) \rightarrow \mathbf{Z}_{\ell}^{\times} \subset \mathbf{Q}_{\ell}^{\times}
$$

denote the cyclotomic character. If $r$ is an integer, then the $\operatorname{Gal}\left(F^{s} / F\right)$-module $\mathbf{Q}_{\ell}(r)$ is the $\mathbf{Q}_{\ell \text {-vector space }} \mathbf{Q}_{\ell}$ with Galois action defined by the character $\chi_{\ell}^{r}$. We have $\mathbf{Q}_{\ell}(r)=\mathbf{Q}(r) \otimes_{\mathbf{Q}} \mathbf{Q}_{\ell}$ (see $\S 1$ of $[$ 国). Suppose $A$ is an abelian variety over $F$. Let $V_{\ell}=V_{\ell}(A)$ and let $V_{\ell}^{*}$ be the dual of $V_{\ell}$. If $\nu \in \mathbf{G}_{m}$, let $\nu$ act on $\mathbf{Q}_{\ell}(1)$ as $\nu^{-1}$. We obtain a canonical action of $G L\left(V_{\ell}\right) \times \mathbf{G}_{m}$ on $V_{\ell}^{\otimes p} \otimes\left(V_{\ell}^{*}\right)^{\otimes q} \otimes \mathbf{Q}_{\ell}(r)$. Define

$$
\tilde{\rho}_{A, \ell}: \operatorname{Gal}\left(F^{s} / F\right) \rightarrow \operatorname{Aut}\left(V_{\ell}\right) \times \mathbf{Q}_{\ell}^{\times}=\operatorname{Aut}\left(V_{\ell}\right) \times \mathbf{G}_{m}\left(\mathbf{Q}_{\ell}\right)
$$

by $\tilde{\rho}_{A, \ell}(\sigma)=\left(\rho_{A, \ell}(\sigma), \chi_{\ell}^{-1}(\sigma)\right)$.

Definition 2.11. Let $\tilde{\mathfrak{G}}_{\ell}(F, A)$ denote the smallest $\mathbf{Q}_{\ell}$-algebraic subgroup of

$$
G L\left(V_{\ell}\right) \times \mathbf{G}_{m}
$$

whose group of $\mathbf{Q}_{\ell}$-points contains the image of $\tilde{\rho}_{A, \ell}$.

If $A$ is a complex abelian variety, then a polarization on $A$ (i.e., the imaginary part of a Riemann form) produces an element $E$ of $\operatorname{Hom}\left(\wedge^{2} V, \mathbf{Q}(1)\right)$ which is a rational tensor of bidegree $(0,0)$. If $A$ is an abelian variety over an arbitrary field $F$, then a polarization on $A$ defined over $F$ defines a $\operatorname{Gal}\left(F^{s} / F\right)$-invariant element $E_{\ell}$ of $\operatorname{Hom}\left(\wedge^{2} V_{\ell}, \mathbf{Q}_{\ell}(1)\right)$ (since the Weil pairing is $\operatorname{Gal}\left(F^{s} / F\right)$-equivariant). If $\bar{F}$ is a subfield of $\mathbf{C}$, and we fix a polarization on $A$ defined over $F$, then the line generated by $E_{\ell}$ in $\operatorname{Hom}\left(\wedge^{2} V_{\ell}, \mathbf{Q}_{\ell}(1)\right)$ is the extension of scalars to $\mathbf{Q}_{\ell}$ of the line generated by $E$ in $\operatorname{Hom}\left(\wedge^{2} V, \mathbf{Q}(1)\right)$. (See p. 237 of [1], especially the last sentence.)

The following result implies that the projection map $G L(V) \times \mathbf{G}_{m} \rightarrow G L(V)$ induces an isomorphism from $\widetilde{M T}_{A}$ onto $M T_{A}$. Since we were not able to find a proof in the literature, we have included one for the benefit of the reader. 
Proposition 2.12. If $A$ is a complex abelian variety, then there exists a (unique) character $\gamma: M T_{A} \rightarrow \mathbf{G}_{m}$ such that $\widetilde{M T}_{A}$ is the graph of $\gamma$.

Proof. Let $p_{1}$ and $p_{2}$ denote the projection maps from $G L(V) \times \mathbf{G}_{m}$ onto $G L(V)$ and $\mathbf{G}_{m}$, respectively. By Lemma 2.10, $M T_{A}$ is the image of $\widetilde{M T}_{A}$ under $p_{1}$. Fix a polarization on $A$. The polarization generates a line $D$ in the $\mathbf{Q}$-vector space $\operatorname{Hom}\left(\wedge^{2} V, \mathbf{Q}(1)\right)$, on which $\widetilde{M T}_{A}$ acts trivially. Let $D(-1)=D \otimes \mathbf{Q}(-1)$, a line in $\operatorname{Hom}\left(\wedge^{2} V, \mathbf{Q}\right)$. Since $\widetilde{M T}_{A}$ acts trivially on $D, \widetilde{M T}_{A}$ acts on $D(-1)$ via $p_{2}$. Let

$$
B=\{\alpha \in G L(V): \alpha D(-1) \subseteq D(-1)\}
$$

and let the character $\gamma: B \rightarrow \operatorname{Aut}(D(-1))=\mathbf{G}_{m}$ be induced by the action of $G L(V)$ on $\operatorname{Hom}\left(\wedge^{2} V, \mathbf{Q}\right)$. The action of $G L(V) \times \mathbf{G}_{m}$ on $\operatorname{Hom}\left(\wedge^{2} V, \mathbf{Q}\right)$ factors through $G L(V)$. Therefore $M T_{A} \subseteq B$, and we have a commutative diagram

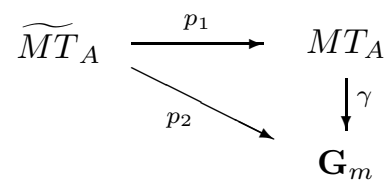

which gives the desired result.

Proposition 2.13. If $A$ is an abelian variety over a field $F, \ell$ is a prime number, and $\ell \neq \operatorname{char}(F)$, then there exists a (unique) character $\gamma_{\ell}: \mathfrak{G}_{\ell}(F, A) \rightarrow \mathbf{G}_{m}$ such that

(i) $\tilde{\mathfrak{G}}_{\ell}(F, A)$ is the graph of $\gamma_{\ell}$,

(ii) the restriction of $\gamma_{\ell}$ to $G_{F, A}$ is $\chi_{\ell}^{-1}$,

(iii) if $\bar{F}$ is a subfield of $\mathbf{C}$, then $\gamma_{\ell}=\gamma$ on $M T_{A, \ell} \cap \mathfrak{G}_{\ell}(F, A)$.

Proof. Let $\pi_{1}$ and $\pi_{2}$ denote the projection maps from $G L\left(V_{\ell}\right) \times \mathbf{G}_{m}$ onto $G L\left(V_{\ell}\right)$ and $\mathbf{G}_{m}$, respectively. By the definitions, $\mathfrak{G}_{\ell}(F, A)$ is the image of $\tilde{\mathfrak{G}}_{\ell}(F, A)$ under $\pi_{1}$. Fix a polarization on $A$ defined over $F$. The polarization generates a line $D_{\ell}$

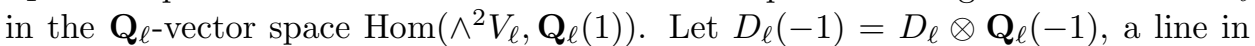
$\operatorname{Hom}\left(\wedge^{2} V_{\ell}, \mathbf{Q}_{\ell}\right)$. Since the Weil pairing is $\operatorname{Gal}\left(F^{s} / F\right)$-equivariant, $\operatorname{Gal}\left(F^{s} / F\right)$ acts trivially on $D_{\ell}$. Therefore $\tilde{\mathfrak{G}}_{\ell}(F, A)$ acts trivially on $D_{\ell}$, and acts via $\pi_{2}$ on $D_{\ell}(-1)$. Let

$$
B_{\ell}=\left\{\alpha \in G L\left(V_{\ell}\right): \alpha D_{\ell}(-1) \subseteq D_{\ell}(-1)\right\}
$$

and let the character $\gamma_{\ell}: B_{\ell} \rightarrow \operatorname{Aut}\left(D_{\ell}(-1)\right)=\mathbf{G}_{m}$ be induced by the action of $G L\left(V_{\ell}\right)$ on $\operatorname{Hom}\left(\wedge^{2} V_{\ell}, \mathbf{Q}_{\ell}\right)$. The action of $G L\left(V_{\ell}\right) \times \mathbf{G}_{m}$ on $\operatorname{Hom}\left(\wedge^{2} V_{\ell}, \mathbf{Q}_{\ell}\right)$ factors through the action of $G L\left(V_{\ell}\right)$. Therefore $\tilde{\mathfrak{G}}_{\ell}(F, A) \subseteq B_{\ell}$, and we have a commutative diagram

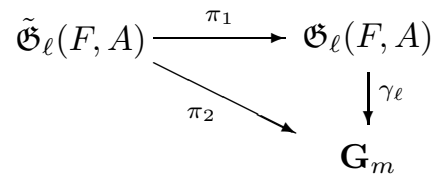

which gives (i). Since the restriction of $\pi_{2}$ to $G_{F, A}$ is $\chi_{\ell}^{-1}$, we have (ii). Now suppose $\bar{F}$ is a subfield of $\mathbf{C}$. Using the fixed polarization, define $D, D(-1), B$, and $\gamma$ as in the proof of Theorem 2.12. Then $B_{\ell}=B \times_{\mathbf{Q}} \mathbf{Q}_{\ell}$, and therefore $M T_{A, \ell} \subseteq B_{\ell}$. Since $\gamma$ 
(respectively, $\gamma_{\ell}$ ) is induced by the action of $G L(V)$ on $\operatorname{Hom}\left(\wedge^{2} V, \mathbf{Q}\right)$ (respectively, $G L\left(V_{\ell}\right)$ on $\left.\operatorname{Hom}\left(\wedge^{2} V_{\ell}, \mathbf{Q}_{\ell}\right)\right)$, and $V_{\ell}=V \otimes_{\mathbf{Q}} \mathbf{Q}_{\ell}$, we have (iii).

Write $\widetilde{M T}_{A, \ell}$ for the $\mathbf{Q}_{\ell}$-algebraic subgroup $\widetilde{M T}_{A} \times{ }_{\mathbf{Q}} \mathbf{Q}_{\ell}$ of $G L\left(V_{\ell}\right) \times \mathbf{G}_{m}$. Then $\widetilde{M T}_{A}\left(\mathbf{Q}_{\ell}\right)=\widetilde{M T}_{A, \ell}\left(\mathbf{Q}_{\ell}\right)$. We state a reformulation of Theorem 2.8, which we will use in $\S 3$.

Theorem 2.14. If $A$ is an abelian variety over a finitely generated extension $F$ of $\mathbf{Q}$, then $\tilde{\mathfrak{G}}_{\ell}(F, A)^{0} \subseteq \widetilde{M T}_{A, \ell}$.

Proof. The result follows directly from Theorem 2.8 and Propositions 2.12 and 2.13 .

\section{Connectedness extensions}

Theorem 3.1. Suppose $A$ is an abelian variety over a field $F, \ell$ is a prime number not equal to $\operatorname{char}(F)$,

$$
c: \operatorname{Gal}\left(F^{s} / F\right) \rightarrow \operatorname{Aut}_{F}(A) \subseteq \operatorname{Aut}\left(V_{\ell}(A)\right)
$$

is a homomorphism, $B$ is the twist of $A$ by the cocycle determined by $c$, and

$$
F=F(\operatorname{End}(A))=F_{\Phi, \ell}(A) .
$$

Then:

(i) c induces an isomorphism

$$
\operatorname{Gal}\left(F_{\Phi, \ell}(B) / F\right) \cong \operatorname{Im}(c) /\left(\operatorname{Im}(c) \cap \mathfrak{G}_{\ell}(F, A)\left(\mathbf{Q}_{\ell}\right)\right),
$$

(ii) $\mathfrak{G}_{\ell}(F, B)$ is connected if and only if $\operatorname{Im}(c) \subseteq \mathfrak{G}_{\ell}(F, A)\left(\mathbf{Q}_{\ell}\right)$,

(iii) if $M$ is the abelian extension of $F$ in $F^{s}$ cut out by $c$, then $c$ induces an isomorphism

$$
\operatorname{Gal}\left(M / F_{\Phi, \ell}(B)\right) \cong \operatorname{Im}(c) \cap \mathfrak{G}_{\ell}(F, A)\left(\mathbf{Q}_{\ell}\right) .
$$

Proof. By Lemma 2.2 ii, $F_{\Phi, \ell}(B) \subseteq M$. The character $c$ induces isomorphisms

$$
\operatorname{Gal}(M / F) \cong \operatorname{Im}(c)
$$

and

$$
\operatorname{Gal}\left(M / F_{\Phi, \ell}(B)\right) \cong \operatorname{Im}(c) \cap \mathfrak{G}_{\ell}(F, B)^{0}\left(\mathbf{Q}_{\ell}\right) .
$$

By Lemma 2.2 , we have $\mathfrak{G}_{\ell}(F, B)^{0} \cong \mathfrak{G}_{\ell}(F, A)$, and the result follows.

Corollary 3.2. Suppose $A$ is an abelian variety over a field $F, \ell$ is a prime number not equal to $\operatorname{char}(F), B$ is the twist of $A$ by a cocycle

$$
c: \operatorname{Gal}\left(F^{s} / F\right) \rightarrow \operatorname{Aut}(A) \subseteq \operatorname{Aut}\left(V_{\ell}(A)\right),
$$

and

$$
F=F(\operatorname{End}(A))=F_{\Phi, \ell}(A)=F(\operatorname{End}(B)) .
$$

Then:

(i) $c$ is a character with values in $Z_{A}^{\times}$(where $Z_{A}$ denotes the center of $\operatorname{End}(A)$ ),

(ii) c induces an isomorphism

$$
\operatorname{Gal}\left(F_{\Phi, \ell}(B) / F\right) \cong \operatorname{Im}(c) /\left(\operatorname{Im}(c) \cap \mathfrak{G}_{\ell}(F, A)\left(\mathbf{Q}_{\ell}\right)\right),
$$

(iii) $\mathfrak{G}_{\ell}(F, B)$ is connected if and only if $\operatorname{Im}(c) \subseteq \mathfrak{G}_{\ell}(F, A)\left(\mathbf{Q}_{\ell}\right)$, 
(iv) if $M$ is the abelian extension of $F$ in $F^{s}$ cut out by $c$, then $c$ induces an isomorphism

$$
\operatorname{Gal}\left(M / F_{\Phi, \ell}(B)\right) \cong \operatorname{Im}(c) \cap \mathfrak{G}_{\ell}(F, A)\left(\mathbf{Q}_{\ell}\right) .
$$

Proof. By Lemma 2.5 and the assumption that $F=F(\operatorname{End}(B))$, we have (i). The result now follows from Theorem 3.1.

Suppose $A$ is an abelian variety defined over a field $F$ of characteristic zero, $k$ is a CM-field, $\iota: k \hookrightarrow \operatorname{End}_{F}^{0}(A)$ is an embedding, and $C$ is an algebraically closed field containing $F$. Let $\operatorname{Lie}(A)$ be the tangent space of $A$ at the origin, an $F$-vector space. If $\sigma$ is an embedding of $k$ into $C$, let

$$
n_{\sigma}=\operatorname{dim}_{C}\left\{t \in \operatorname{Lie}(A) \otimes_{F} C: \iota(\alpha) t=\sigma(\alpha) t \text { for all } \alpha \in k\right\} .
$$

Write $\bar{\sigma}$ for the composition of $\sigma$ with the involution complex conjugation of $k$.

Definition 3.3. If $A$ is an abelian variety over an algebraically closed field $C$ of characteristic zero, $k$ is a CM-field, and $\iota: k \hookrightarrow \operatorname{End}^{0}(A)$ is an embedding, we say $(A, k, \iota)$ is of Weil type if $n_{\sigma}=n_{\bar{\sigma}}$ for all embeddings $\sigma$ of $k$ into $C$.

Although we do not use this fact, we remark that $(A, k, \iota)$ is of Weil type if and only if $\iota$ makes $\operatorname{Lie}(A) \otimes_{F} C$ into a free $k \otimes_{\mathbf{Q}} C$-module (see p. 525 of 13 for the case where $k$ is an imaginary quadratic field). Using the semisimplicity of the $F$-algebra $k \otimes_{\mathbf{Q}} F$ and the $C$-algebra $k \otimes_{\mathbf{Q}} C$, one may easily deduce that $\iota$ makes $\operatorname{Lie}(A) \otimes_{F} C$ into a free $k \otimes_{\mathbf{Q}} C$-module if and only if $\iota$ makes $\operatorname{Lie}(A)$ into a free $k \otimes_{\mathbf{Q}} F$-module.

Suppose $(A, k, \iota)$ is of Weil type, and we have an element of

$$
H^{1}(\operatorname{Gal}(\bar{F} / F), \operatorname{Aut}(A))
$$

which is represented by a cocycle $c$ with values in the center of $\operatorname{End}^{0}(A)$. Let $B$ be the twist of $A$ by $c$, and let $\varphi$ be the isomorphism from $\operatorname{End}_{F}(A)$ to $\operatorname{End}_{F}(B)$ obtained in Corollary 2.4 and Proposition 2.3. Since $(A, \iota)$ and $(B, \varphi \circ \iota)$ are isomorphic over $C$, it follows that $(B, k, \varphi \circ \iota)$ is of Weil type.

Note that if $(A, k, \iota)$ is of Weil type, then $\operatorname{dim}(A)$ is divisible by $[k: \mathbf{Q}]$.

Theorem 3.4. Suppose $A$ is an abelian variety over a finitely generated extension $F$ of $\mathbf{Q}, \ell$ is a prime number, $k$ is a CM-field, $\iota$ is an embedding of $k$ into the center of $\operatorname{End}^{0}(A)$ such that $(A, k, \iota)$ is of Weil type, $c: \operatorname{Gal}(\bar{F} / F) \rightarrow k^{\times}$is a character of finite order $n, r=2 \operatorname{dim}(A) /[k: \mathbf{Q}] \in \mathbf{Z}, M$ is the $\mathbf{Z} / n \mathbf{Z}$-extension of $F$ cut out by $c$, and $B$ is the twist of $A$ by $c$. Suppose $F=F(\operatorname{End}(A))$, $\iota \circ c$ takes values in $\operatorname{Aut}(A), r$ is even, and $n$ does not divide $r$. Then

(i) $F=F(\operatorname{End}(B))$,

(ii) either $F \neq F_{\Phi}(A)$ or $F \neq F_{\Phi}(B)$,

(iii) if $F_{\Phi}(A)=F$, then $F_{\Phi}(B) \subseteq M$ and $\left[M: F_{\Phi}(B)\right]$ divides $(n, 2 r)$,

(iv) if $F_{\Phi}(A)=F$ and $(n, 2 r)=2$, then $\left[M: F_{\Phi}(B)\right]=2$.

Proof. The Galois module $V_{\ell}(B)$ is the twist of $V_{\ell}(A)$ by $c$ (see Remark 2.6). By applying Corollary 2.4 to the cocycle induced by $c$, we deduce (i) and we obtain an isomorphism $\varphi$ from $\operatorname{End}_{F}(A)$ onto $\operatorname{End}_{F}(B)$ such that $(B, k, \varphi \circ \iota)$ is of Weil type. Let $k_{\ell}=k \otimes \mathbf{Q}_{\ell}$. For $U=A$ or $B$, let

$$
W_{U}=\operatorname{Hom}_{\mathbf{Q}}\left(\wedge_{k}^{r} H_{1}(U, \mathbf{Q}), \mathbf{Q}\left(\frac{r}{2}\right)\right), \quad W_{U, \ell}=\operatorname{Hom}_{\mathbf{Q}_{\ell}}\left(\wedge_{k_{\ell}}^{r} V_{\ell}(U), \mathbf{Q}_{\ell}\left(\frac{r}{2}\right)\right),
$$

where $\operatorname{Hom}_{E}$ means homomorphisms of $E$-vector spaces, if $E$ is a field. Then $W_{U}$ is a one-dimensional $k$-vector space and $W_{U, \ell}$ is a free rank-one $k_{\ell}$-module. The 
elements of $W_{U}$ are called Weil classes for $U$. Since $V_{\ell}(U)=H_{1}(U, \mathbf{Q}) \otimes_{\mathbf{Q}} \mathbf{Q}_{\ell}$, we have $W_{U, \ell}=W_{U} \otimes_{\mathbf{Q}} \mathbf{Q}_{\ell}$. Consider the action of the Galois group $\operatorname{Gal}(\bar{F} / F)$. The Galois module $W_{B, \ell}$ is the twist of the Galois module $W_{A, \ell}$ by the character $c^{-r}$. Since $n$ does not divide $r$, this is a non-trivial twist, so the Galois modules $W_{B, \ell}$ and $W_{A, \ell}$ cannot be simultaneously trivial.

By pp. 52-54 of [4] (see also Lemma 2.8 of [10] and p. 423 of [25]), the elements of $W_{U}$ are Hodge classes (since we are dealing with abelian varieties of Weil type). Since $\widetilde{M} T_{U}$ acts trivially on the Hodge classes, $\widetilde{M} T_{U, \ell}\left(\mathbf{Q}_{\ell}\right)$ acts trivially on $W_{U, \ell}=$ $W_{U} \otimes_{\mathbf{Q}} \mathbf{Q}_{\ell}$. Suppose now that $\mathfrak{G}_{\ell}(F, A)$ and $\mathfrak{G}_{\ell}(F, B)$ are both connected. Then $\tilde{\mathfrak{G}}_{\ell}(F, A)$ and $\tilde{\mathfrak{G}}_{\ell}(F, B)$ are both connected (by Proposition 2.13 i). It follows from Theorem 2.14 that $\tilde{\mathfrak{G}}_{\ell}(F, U) \subseteq \widetilde{M} T_{U, \ell}$. Therefore, $W_{B, \ell}$ and $W_{A, \ell}$ are both trivial as $\operatorname{Gal}(\bar{F} / F)$-modules. This is a contradiction. We therefore have (ii).

Suppose that $F_{\Phi}(A)=F$. Then $\mathfrak{G}_{\ell}(F, A)$ is connected, so $\mathfrak{G}_{\ell}(F, B)$ is disconnected. By Lemma 2.2, $\mathfrak{G}_{\ell}(M, B)$ is connected. Therefore, $F_{\Phi}(B) \subseteq M$. By Corollary $3.2 \mathrm{v}$,

$$
\operatorname{Gal}\left(M / F_{\Phi}(B)\right) \cong \operatorname{Im}(c) \cap \mathfrak{G}_{\ell}(F, A)\left(\mathbf{Q}_{\ell}\right)
$$

Let $\mu_{s}(k)$ denote the group of $s$-th roots of unity in $k^{\times}$. We have

$$
\operatorname{Im}(c)=\mu_{n}(k) \cong \mathbf{Z} / n \mathbf{Z}
$$

Suppose $\alpha \in \operatorname{Im}(c) \cap \mathfrak{G}_{\ell}(F, A)\left(\mathbf{Q}_{\ell}\right)$. Then $\alpha^{n}=1$. By Theorem 2.8 and the facts that $\mathfrak{G}_{\ell}(F, A)=\mathfrak{G}_{\ell}(F, A)^{0}$ and $\alpha \in \operatorname{End}^{0}(A)$, we have $\alpha \in M T_{A}(\mathbf{Q})$. Applying the character $\gamma$ of Theorem 2.12, we have that $\gamma(\alpha)$ is an $n$-th root of unity in $\mathbf{Q}^{\times}$, and therefore $\gamma(\alpha)$ is 1 or -1 . By the definition of $W_{A, \ell}, \alpha$ acts on $W_{A, \ell}$ as multiplication by $\alpha^{-r} \gamma(\alpha)^{-r / 2}$. Since $\alpha \in M T_{A, \ell}\left(\mathbf{Q}_{\ell}\right), \alpha$ acts trivially on $W_{A, \ell}$. Therefore $\alpha^{-r} \gamma(\alpha)^{-r / 2}=1$, so $\alpha^{2 r}=1$. Let $t=(n, 2 r)$. Then

$$
\operatorname{Gal}\left(M / F_{\Phi}(B)\right) \cong \operatorname{Im}(c) \cap \mathfrak{G}_{\ell}(F, A)\left(\mathbf{Q}_{\ell}\right) \subseteq \mu_{n}(k) \cap \mu_{2 r}(k)=\mu_{t}(k) .
$$

Therefore, $\left[M: F_{\Phi}(B)\right]$ divides $t$. Since $\mathfrak{G}_{\ell}(F, A)$ contains the homotheties $\mathbf{G}_{m}$ (see 2.3 of $\sqrt{14})$, we have $-1 \in \mathfrak{G}_{\ell}(F, A)\left(\mathbf{Q}_{\ell}\right)$. So $-1 \in \operatorname{Im}(c) \cap \mathfrak{G}_{\ell}(F, A)\left(\mathbf{Q}_{\ell}\right)$ if and only if $-1 \in \operatorname{Im}(c)$, i.e., if and only if $n$ is even. Thus if $t=2$, then

$$
\operatorname{Gal}\left(M / F_{\Phi}(B)\right) \cong\{ \pm 1\}
$$

Theorem 3.5. Suppose $X$ and $Y$ are abelian varieties over a finitely generated extension $F$ of $\mathbf{Q}, \ell$ is a prime number, $\operatorname{Hom}(X, Y)=0, F=F(\operatorname{End}(X))=$ $F(\operatorname{End}(Y)), k$ is a $C M$-field, $[k: \mathbf{Q}]=2 \operatorname{dim}(Y)$, and $\operatorname{dim}(X)=t \operatorname{dim}(Y)$ for some odd positive integer $t$. Suppose $\iota_{X}$ and $\iota_{Y}$ are embeddings of $k$ into $\operatorname{End}^{0}(X)$ and $\operatorname{End}^{0}(Y)$, respectively, and $\left(X \times Y, k, \iota_{X} \times \iota_{Y}\right)$ is of Weil type. Suppose $c$ is the non-trivial character associated to a quadratic extension $M$ of $F$, let $Y^{c}$ denote the twist of $Y$ by $c$, let $A=X \times Y$, and let $B=X \times Y^{c}$. Then

(i) $F=F(\operatorname{End}(B))$,

(ii) either $F(\operatorname{End}(A)) \neq F_{\Phi}(A)$ or $F(\operatorname{End}(B)) \neq F_{\Phi}(B)$,

(iii) if $F_{\Phi}(A)=F$, then $F_{\Phi}(B)=M$.

Proof. We have $F=F(\operatorname{End}(A))$. Since $\operatorname{Hom}(X, Y)=0$, we have $\operatorname{End}^{0}(A)=$ $\operatorname{End}^{0}(X) \oplus \operatorname{End}^{0}(Y)$ and $\operatorname{Aut}(A)=\operatorname{Aut}(X) \times \operatorname{Aut}(Y)$. Consider the cocycle $c$ that sends $\sigma \in \operatorname{Gal}(\bar{F} / F)$ to $(1, c(\sigma)) \in \operatorname{Aut}(X) \times \operatorname{Aut}(Y)=\operatorname{Aut}(A)$. All the values of $c$ are of the form $(1, \pm 1)$, and therefore belong to the center of $\operatorname{End}^{0}(A)$. The abelian 
variety $B\left(=X \times Y^{c}\right)$ is the twist of $A(=X \times Y)$ by $c$. By Corollary 2.4 we have (i), and we obtain an isomorphism $\varphi$ from $\operatorname{End}_{F}(A)$ onto $\operatorname{End}_{F}(B)$ such that $\left(B, k, \varphi \circ\left(\iota_{X} \times \iota_{Y}\right)\right)$ is of Weil type. Let $k_{\ell}=k \otimes \mathbf{Q}_{\ell}$. We have

$$
V_{\ell}(A)=V_{\ell}(X) \oplus V_{\ell}(Y), \quad V_{\ell}(B)=V_{\ell}(X) \oplus V_{\ell}\left(Y^{c}\right) .
$$

Viewing the Tate modules as free $k_{\ell}$-modules, we have

$$
\wedge_{k_{\ell}}^{t+1} V_{\ell}(A)=\wedge_{k_{\ell}}^{t} V_{\ell}(X) \otimes_{k_{\ell}} V_{\ell}(Y), \quad \wedge_{k_{\ell}}^{t+1} V_{\ell}(B)=\wedge_{k_{\ell}}^{t} V_{\ell}(X) \otimes_{k_{\ell}} V_{\ell}\left(Y^{c}\right) .
$$

For $U=A$ or $B$, let

$$
W_{U, \ell}=\operatorname{Hom}_{\mathbf{Q}_{\ell}}\left(\wedge_{k_{\ell}}^{t+1} V_{\ell}(U), \mathbf{Q}_{\ell}\left(\frac{t+1}{2}\right)\right)
$$

The Galois module $V_{\ell}\left(Y^{c}\right)$ is the twist of the Galois module $V_{\ell}(Y)$ by the character $c$ (see Remark 2.6), and the Galois module $W_{B, \ell}$ is the twist of the Galois module $W_{A, \ell}$ by $c^{-1}=c$. Since $c$ is non-trivial, the Galois modules $W_{B, \ell}$ and $W_{A, \ell}$ cannot be simultaneously trivial. As in the proof of Theorem 3.4, it follows that $\mathfrak{G}_{\ell}(F, A)$ and $\mathfrak{G}_{\ell}(F, B)$ cannot both be connected. If $F_{\Phi}(A)=F$, then $\mathfrak{G}_{\ell}(F, A)$ is connected, so $\mathfrak{G}_{\ell}(F, B)$ is disconnected. By Lemma 2.2, $\mathfrak{G}_{\ell}(M, B)$ is connected, and so $F_{\Phi}(B)$ must be the quadratic extension $M$ of $F$.

Remark 3.6. Suppose $F$ is a subfield of $\mathbf{C}, Y$ is an elliptic curve over $F$ with complex multiplication by an imaginary quadratic field $K$, and $X$ is an absolutely simple 3-dimensional abelian variety over $F$ with $K$ embedded in its endomorphism algebra. Then we can always ensure (by taking complex conjugates if necessary) that the two embeddings of $K$ into $\mathbf{C}$ occur with the same multiplicity in the action of $K$ on the tangent space of the 4-dimensional abelian variety $A=X \times Y$. Note that the hypotheses of Theorem 3.5 (or of Theorem 3.4) cannot be simultaneously satisfied with $\operatorname{dim}(A)<4$. In Example 4.2 we exhibit 4 -dimensional abelian varieties satisfying the hypotheses of Theorem 3.5 .

\section{EXAmples}

Using Theorems 3.4 and 3.5, we can construct examples of abelian varieties $B$ such that $\mathfrak{G}_{\ell}(F, B)$ is disconnected, and compute the connectedness extensions.

4.1. Example. Let $k=\mathbf{Q}(\sqrt{-3})$ and let $K$ be the CM-field which is the compositum of $\mathbf{Q}(\sqrt{-3})$ with the maximal totally real subfield $L$ of $\mathbf{Q}\left(\zeta_{17}\right)$. Then

$$
\operatorname{Gal}(K / \mathbf{Q}) \cong \operatorname{Gal}(k / \mathbf{Q}) \times \operatorname{Gal}(L / \mathbf{Q}) \cong \mathbf{Z} / 2 \mathbf{Z} \times \mathbf{Z} / 8 \mathbf{Z} .
$$

Let $\Psi$ be the subset of $\mathbf{Z} / 2 \mathbf{Z} \times \mathbf{Z} / 8 \mathbf{Z} \cong \operatorname{Gal}(K / \mathbf{Q})$ defined by

$$
\Psi=\{(0,0),(0,1),(0,4),(0,7),(1,2),(1,3),(1,5),(1,6)\} .
$$

Let $\mathcal{O}_{K}$ denote the ring of integers of $K$. Let $\left(A, \iota_{K}\right)$ be an 8-dimensional CM abelian variety of CM-type $(K, \Psi)$ constructed from the lattice $\mathcal{O}_{K}$ as in Theorem 3 on p. 46 of [20], and defined over a number field $F$ (this can be done by Proposition 26 on p. 109 of 20]). Then $A$ is absolutely simple, by the choice of $\Psi$ and Proposition 26 on p. 69 of 20], and $\operatorname{End}(A)=\mathcal{O}_{K}$ (see Proposition 6 on p. 42 of 20]). Further, the reflex field of $(K, \Psi)$ is $K$. Take the number field $F$ to be sufficiently large so that $F_{\Phi}(A)=F$. Let $\iota$ be the restriction of $\iota_{K}$ to $k$. By the definition of $\Psi$, if $\sigma \in \operatorname{Gal}(k / \mathbf{Q})=\operatorname{Hom}(k, \mathbf{C})$ then $n_{\sigma}=4$. Therefore $(A, k, \iota)$ is of Weil type. Let $c: \operatorname{Gal}(\bar{F} / F) \rightarrow k^{\times}$be a non-trivial cubic character associated to a cubic extension 
$M$ of $F$, and let $B$ denote the twist of $A$ by $c$. Applying Theorem 3.4 iii with $n=3$ and $r=8$, then $F(\operatorname{End}(B))=F$ and $F_{\Phi}(B)=M$.

4.2. Example. Let $J$ be the Jacobian of the genus 3 curve

$$
y^{7}=x(1-x),
$$

and let $E$ be the elliptic curve $X_{0}(49)$. A model for $E$ is given by the equation

$$
y^{2}+x y=x^{3}-x^{2}-2 x-1 .
$$

Let $d$ be a non-zero square-free integer. If $d \neq 1$ let $E^{(d)}$ be the twist of $E$ by the non-trivial character of $\mathbf{Q}(\sqrt{d})$, and if $d=1$ let $E^{(d)}=E$. Let

$$
A=J \times E, \quad A^{(d)}=J \times E^{(d)} .
$$

The abelian varieties $A^{(d)}$ are defined over $\mathbf{Q}$. Let $\zeta_{7}$ be a primitive seventh root of unity and let

$$
K=\mathbf{Q}\left(\zeta_{7}\right), \quad L_{d}=K(\sqrt{d}), \quad \text { and } \quad k=\mathbf{Q}(\sqrt{-7}) .
$$

If $d=1$ or -7 then $K=L_{d}$; otherwise, $\left[L_{d}: K\right]=2$. The abelian variety $J$ is a simple abelian variety with complex multiplication by $K$, and the elliptic curves $E^{(d)}$ have complex multiplication by the subfield $k$ of $K$. We have $\operatorname{Gal}(K / \mathbf{Q})=$ $\left\{\sigma_{1}, \ldots, \sigma_{6}\right\}$ where $\sigma_{i}\left(\zeta_{7}\right)=\zeta_{7}^{i}$. The CM-type of $J$ is $\left(K,\left\{\sigma_{1}, \sigma_{2}, \sigma_{3}\right\}\right)$ (see p. 34 of [5] or $\S 15.4 .2$ of [20]), and the reflex CM-type is $\left(K,\left\{\sigma_{4}, \sigma_{5}, \sigma_{6}\right\}\right)$ (see $\S 8.4 .1$ of 20]). We can identify $\operatorname{End}\left(A^{(d)}\right)$ with the direct sum of $\operatorname{End}(J)$ and $\operatorname{End}\left(E^{(d)}\right)$. By Proposition 30 on p. 74 of [20], the smallest extension of $\mathbf{Q}$ over which all the elements of $\operatorname{End}(J)$ are defined is the reflex CM-field of the CM-type of $J$, which is $K$. Similarly, $k$ is the smallest extension of $\mathbf{Q}$ over which all the elements of $\operatorname{End}\left(E^{(d)}\right)$ are defined. We therefore have

$$
K=\mathbf{Q}\left(\operatorname{End}\left(A^{(d)}\right)\right) .
$$

Next, we will prove that $L_{d}=\mathbf{Q}_{\Phi}\left(A^{(d)}\right)$.

Write $\mathcal{O}_{\Omega}$ for the ring of integers of a number field $\Omega$. If $q$ is a prime number, let $\mathcal{O}_{q}=\mathcal{O}_{\Omega} \otimes \mathbf{Z}_{q}$.

Lemma 4.2.1. If $K^{\prime}$ is a finite abelian extension of $K$ which is unramified away from the primes above 7 , then $\left[K^{\prime}: K\right]$ is a power of 7 .

Proof. We have $-1-\zeta_{7}=\left(1-\zeta_{7}^{2}\right) /\left(\zeta_{7}-1\right) \in \mathcal{O}_{K}^{\times}$. Let $\mathcal{P}$ be the prime ideal of $K$ above 7 . The reduction map

$$
\mathcal{O}_{K}^{\times} \rightarrow\left(\mathcal{O}_{K} / \mathcal{P}\right)^{\times} \cong(\mathbf{Z} / 7 \mathbf{Z})^{\times}
$$

is surjective, since $-1-\zeta_{7}$ maps to -2 , a generator of $(\mathbf{Z} / 7 \mathbf{Z})^{\times}$. Moreover, the class number of $K$ is one. Therefore by class field theory, there is no non-trivial abelian extension of $K$ of degree prime to 7 and unramified away from the primes above $\mathcal{P}$.

Lemma 4.2.2. If $p$ is a prime and $p \equiv 3(\bmod 7)$, then the only field $K^{\prime}$ such that

(i) $K \subseteq K^{\prime} \subseteq K\left(A_{p}\right)$, and

(ii) $K^{\prime} / K$ is unramified away from the primes above 7 , is $K$ itself. 
Proof. Since $K$ is a field of definition for the endomorphisms of the CM abelian varieties $J$ and $E$, the extension $K\left(A_{n}\right) / K$ is abelian for every integer $n$ (see Corollary 2 on p. 502 of $[18]$ ). Suppose $p$ and $K^{\prime}$ satisfy the hypotheses of Lemma 4.2.2. Let $I_{p} \subseteq \operatorname{Gal}\left(K\left(J_{p}\right) / K\right)$ be the inertia subgroup at $p$. We will first show

$$
\#\left(I_{p}\right)=\frac{p^{6}-1}{p^{2}+p+1} .
$$

The image of $\mathcal{O}_{p}^{\times}$in $\operatorname{Gal}\left(K\left(J_{p}\right) / K\right)$ under the Artin map of class field theory is $I_{p}$, and we have natural homomorphisms

$$
\operatorname{Gal}\left(K\left(J_{p}\right) / K\right) \hookrightarrow \operatorname{Aut}_{\mathcal{O}_{K}}\left(J_{p}\right) \cong\left(\mathcal{O}_{K} / p \mathcal{O}_{K}\right)^{\times} \cong \mathcal{O}_{p}^{\times} /\left(1+p \mathcal{O}_{p}\right) .
$$

We therefore obtain maps

$$
\mathcal{O}_{p}^{\times} \rightarrow I_{p} \hookrightarrow \mathcal{O}_{p}^{\times} /\left(1+p \mathcal{O}_{p}\right) .
$$

Since the first map of (2) is surjective, the order of $I_{p}$ is the order of the image of the composition. Since $p \equiv 3(\bmod 7)$, we know that $p$ is inert in $K / \mathbf{Q}$, so $\left(\mathcal{O}_{K} / p \mathcal{O}_{K}\right)^{\times}\left(\cong \mathcal{O}_{p}^{\times} /\left(1+p \mathcal{O}_{p}\right)\right)$ is a cyclic group of order $p^{6}-1$. Since the greatest common divisor of $p^{6}-1$ and $p^{3}\left(p^{2}+p+1\right)$ is $p^{2}+p+1$, equation (11) will be proved when we show that the composition of maps in (2) sends $u \in \mathcal{O}_{p}^{\times}$to $u^{-p^{3}\left(p^{2}+p+1\right)}$ $\left(\bmod 1+p \mathcal{O}_{p}\right)$. We can view elements of $\operatorname{Gal}(K / \mathbf{Q})$ as automorphisms of $\mathcal{O}_{p}^{\times}$. Proposition 7.40 on p. 211 of [19] implies that the image of $u$ is of the form $\alpha(u) / \eta(u)$ $\left(\bmod 1+p \mathcal{O}_{p}\right)$ where $\eta(u)=\sigma_{4}(u) \sigma_{5}(u) \sigma_{6}(u)$ and $\alpha(u) \in K^{\times}$. Write $K_{\mathbf{A}}^{\times}$for the idele group of $K$, and for each archimedean prime $\lambda$ of $K$, define a Grössencharacter $\psi_{\lambda}: K_{\mathbf{A}}^{\times} \rightarrow \mathbf{C}^{\times}$by $\psi_{\lambda}(x)=(\alpha(x) / \eta(x))_{\lambda}$. View $\mathcal{O}_{p}^{\times}$as a subgroup of $K_{\mathbf{A}}^{\times}$. Since $J$ has good reduction outside 7 , we have $\psi_{\lambda}\left(\mathcal{O}_{p}^{\times}\right)=1$, by Theorem 7.42 of [19]. For $u \in \mathcal{O}_{p}^{\times}$, we have $1=\psi_{\lambda}(u)=\alpha(u)_{\lambda}=\alpha(u)$. Therefore the image of $u$ in $\mathcal{O}_{p}^{\times} /\left(1+p \mathcal{O}_{p}\right)$ is $1 / \eta(u)\left(\bmod 1+p \mathcal{O}_{p}\right)$. Since $p$ is inert in $K / \mathbf{Q}$, we have $\operatorname{Gal}(K / \mathbf{Q}) \cong \operatorname{Gal}\left(\left(\mathcal{O}_{K} / p\right) /(\mathbf{Z} / p)\right)=D_{p}$, where $D_{p}$ is the decomposition group at $p$. The latter group is a cyclic group of order 6 generated by the Frobenius element, and we compute that

$$
\sigma_{4}(u) \equiv u^{p^{4}}, \quad \sigma_{5}(u) \equiv u^{p^{5}}, \quad \text { and } \quad \sigma_{6}(u) \equiv u^{p^{3}} \quad\left(\bmod 1+p \mathcal{O}_{p}\right)
$$

$\left(\right.$ since $p^{4} \equiv 4(\bmod 7), p^{5} \equiv 5(\bmod 7)$, and $\left.p^{3} \equiv 6(\bmod 7)\right)$. Therefore

$$
1 / \eta(u) \equiv u^{-p^{3}\left(p^{2}+p+1\right)} \quad\left(\bmod 1+p \mathcal{O}_{p}\right),
$$

as desired.

We have

$$
\operatorname{Gal}\left(K\left(E_{p}\right) / K\right) \hookrightarrow \operatorname{Aut}_{\mathcal{O}_{k}}\left(E_{p}\right) \cong\left(\mathcal{O}_{k} / p \mathcal{O}_{k}\right)^{\times} .
$$

The order of $\left(\mathcal{O}_{k} / p \mathcal{O}_{k}\right)^{\times}$is $p^{2}-1$, which is not divisible by 7 . Therefore $\left[K\left(A_{p}\right)\right.$ : $\left.K\left(J_{p}\right)\right]$ is not divisible by 7 . By Lemma 4.2.1, $\left[K^{\prime}: K\right]$ is a power of 7 . Therefore $K^{\prime} \subseteq K\left(J_{p}\right)$. Since $K^{\prime} / K$ is unramified at $p$, we have $I_{p} \subseteq \operatorname{Gal}\left(K\left(J_{p}\right) / K^{\prime}\right)$. Suppose $K^{\prime} \neq K$. Then \#(I $)$ divides $\left(p^{6}-1\right) / 7$. By (1) $),\left(p^{6}-1\right) /\left(p^{2}+p+1\right)$ divides $\left(p^{6}-1\right) / 7$. Therefore 7 divides $p^{2}+p+1$, which contradicts the assumption that $p \equiv 3(\bmod 7)$. Therefore, $K^{\prime}=K$.

Suppose $p$ and $q$ are distinct odd primes, and $p \equiv 3(\bmod 7)$. Let $K^{\prime}=K\left(A_{p}\right) \cap$ $K\left(A_{q}\right)$. Since $A$ has good reduction outside 7 , the extension $K^{\prime} / K$ is unramified 
away from the primes above 7. By Lemma 4.2.2, we have $K^{\prime}=K$. As mentioned in the introduction, for every integer $n \geq 3$ we have

$$
K_{\Phi}(A) \subseteq K\left(A_{n}\right) .
$$

We therefore obtain

$$
K=K_{\Phi}(A)=\mathbf{Q}_{\Phi}(A) .
$$

It follows from Theorem 3.5 that

$$
L_{d}=\mathbf{Q}_{\Phi}\left(A^{(d)}\right)
$$

Note that Shioda (see Theorem 4.4 of [21]) proved the Hodge Conjecture for $A$, and therefore also for $A^{(d)}$. Thus, the Weil classes on $A^{(d)}$ are algebraic. It follows easily that $L_{d}$ is the smallest extension of $\mathbf{Q}$ over which all the algebraic cycle classes on all powers of $A^{(d)}$ are defined.

Remark 4.2.3. If $A$ is an abelian variety over a finitely generated extension $F$ of $\mathbf{Q}$, and if the (as yet unproved) Tate Conjecture is true for all powers of $A$ over $F_{\Phi}(A)$, then the field $F_{\Phi}(A)$ is the smallest extension of $F$ over which all the algebraic cycle classes on all powers of $A$ are defined.

\section{REFERENCES}

[1] Borovoi, M.: The action of the Galois group on the rational cohomology classes of type $(p, p)$ of abelian varieties (Russian). Mat. Sbornik (N. S.) 94 (136), 649-652 (1974) = Math. USSR Sbornik 23, 613-616 (1974)

[2] Borovoi, M.: The Shimura-Deligne schemes $M_{\mathbf{C}}(G, h)$ and the rational cohomology classes of type $(p, p)$ of abelian varieties (Russian). In: Problems of group theory and homological algebra (Russian) (No. 1, pp. 3-53) Yaroslavl': Yaroslav. Gos. Univ. 1977

[3] Chi, W.: $\ell$-adic and $\lambda$-adic representations associated to abelian varieties defined over number fields. Amer. J. Math. 114, 315-353 (1992)

[4] Deligne, P. (notes by J. Milne): Hodge cycles on abelian varieties. In: P. Deligne, et al.: Hodge cycles, motives, and Shimura varieties (Lecture Notes in Mathematics, vol. 900, pp. 9-100) Berlin Heidelberg New York: Springer 1982

[5] Lang, S.: Complex Multiplication (Grundlehren Math. Wiss. Bd. 255) New York Berlin Heidelberg Tokyo: Springer 1983

[6] Larsen, M., Pink, R.: On $\ell$-independence of algebraic monodromy groups in compatible systems of representations. Invent. math. 107, 603-636 (1992)

[7] Larsen, M., Pink, R.: Abelian varieties, $\ell$-adic representations, and $\ell$-independence. Math. Ann. 302, 561-579 (1995)

[8] Larsen, M., Pink, R.: A connectedness criterion for $\ell$-adic representations. To appear in Israel J. Math.

[9] Milne, J. S.: Shimura varieties and motives. In: U. Jannsen et al.: Motives (Proc. Symp. Pure Math. vol. 55 , Part 2, pp. 447-523) Providence: Amer. Math. Soc. 1994

[10] Moonen, B., Zarhin, Yu. G.: Hodge classes and Tate classes on simple abelian fourfolds. Duke Math. J. 77, 553-581 (1995)

[11] Mumford, D.: Abelian varieties, Second Edition (Tata Lecture Notes) London: Oxford Univ. Press 1974

[12] Piatetski-Shapiro, I. I.: Interrelations between the Tate and Hodge conjectures for abelian varieties (Russian), Mat. Sbornik 85, 610-620 (1971) = Math. USSR Sbornik 14, 615-625 (1971)

[13] Ribet, K.: Hodge classes on certain types of abelian varieties. Amer. J. Math. 105, 523-538 (1983)

[14] Serre, J-P.: Représentations $\ell$-adiques. In: S. Iyanaga: Algebraic Number Theory (Proceedings of the International Taniguchi Symposium, Kyoto, 1976) (pp. 177-193) Tokyo: Japan Society for the Promotion of Science $1977=$ \# 112 of (Euvres (Vol. III, pp. 384-400) Berlin Heidelberg New York Tokyo: Springer 1986

[15] Serre, J-P.: Letters to K. Ribet, Jan. 1, 1981 and Jan. 29, 1981 
[16] Serre, J-P.: Résumé des cours de 1984-1985, Résumé des cours de 1985-1986, Collège de France

[17] Serre, J-P.: Propriétés conjecturales des groupes de Galois motiviques et des représentations $\ell$-adiques. In: U. Jannsen et al.: Motives (Proc. Symp. Pure Math. vol. 55, Part 2, pp. 377400) Providence: Amer. Math. Soc. 1994

[18] Serre, J-P., Tate, J.: Good reduction of abelian varieties. Ann. of Math. 88, 492-517 (1968)

[19] Shimura, G.: Introduction to the arithmetic theory of automorphic functions, Princeton: Princeton Univ. Press 1971

[20] Shimura, G., Taniyama, Y.: Complex multiplication of abelian varieties and its applications to number theory (no. 6) Publ. Math. Soc. Japan 1961

[21] Shioda, T.: Algebraic cycles on abelian varieties of Fermat type. Math. Ann. 258, 65-80 (1981)

[22] Silverberg, A., Zarhin, Yu. G.: Isogenies of abelian varieties. J. Pure and Applied Algebra 90, 23-37 (1993)

[23] Silverberg, A., Zarhin, Yu. G.: Connectedness results for $\ell$-adic representations associated to abelian varieties. Comp. math. 97, 273-284 (1995)

[24] Silverberg, A., Zarhin, Yu. G.: Images of $\ell$-adic representations and automorphisms of abelian varieties. Preprint.

[25] Weil, A.: Abelian varieties and the Hodge ring [1977c]. CEuvres scientifiques (Vol. III, pp. 421429) New York Heidelberg Berlin: Springer 1979

[26] Zarhin, Yu. G.: Weights of simple Lie algebras in the cohomology of algebraic varieties (Russian). Izv. Akad. Nauk SSSR Ser. Mat. 48, 264-304 (1984) = Math. USSR - Izv. 24, $245-282(1985)$

Department of Mathematics, Ohio State University, 231 W. 18 Avenue, Columbus, Онго 43210-1174, USA

E-mail address: silver@math.ohio-state.edu

Department of Mathematics, Pennsylvania State University, University Park, PA 16802, USA,

Institute for Mathematical Problems in Biology, Russian Academy of Sciences, PushChino, Moscow Region, 142292, Russia

E-mail address: zarhin@math.psu.edu 